\title{
LA-UR-017. $=651$
}

Approved for public release;

distribution is unlimited.

Title:

A LOGIC MODEL APPROACH TO CONCEPTUAL DESIGN OF A SCIENTIFIC/INDUSTRIAL COMPLEX

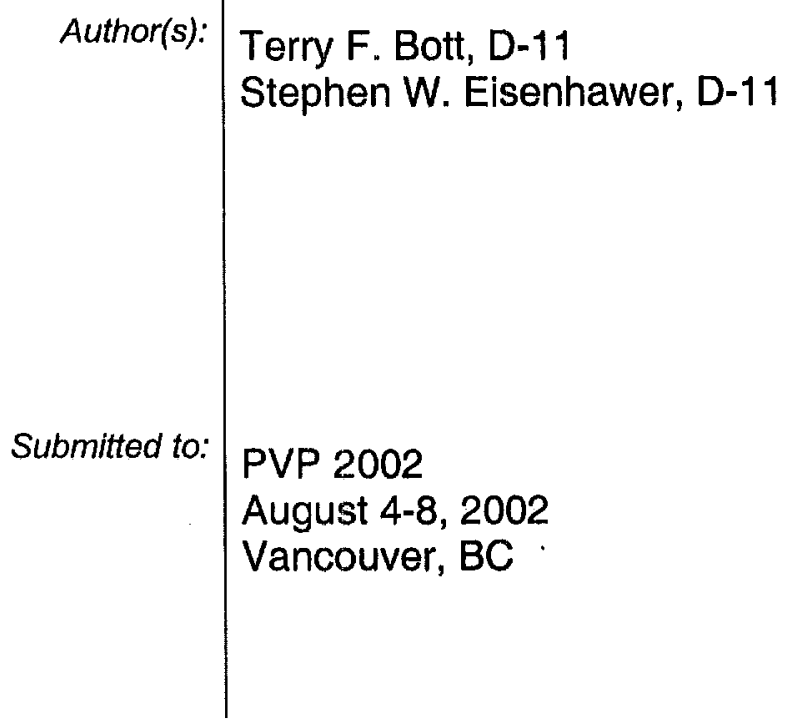

\section{Los Alamos}

NATIONAL. LABORATORY

Los Alamos National Laboratory, an affirmative action/equal opportunity employer, is operated by the University of California for the U.S. Department of Energy under contract W-7405-ENG-36. By acceptance of this article, the publisherregcognizes that the U.S. Government retains a nonexclusive, royalty-free license to publish or reproduce the published form of this cor $\equiv$ tion, or to allow others to do so, for U.S. Government purposes. Los Alamos National Laboratory requests that the publisher identify this le as work performed under the auspices of the U.S. Department of Energy. Los Alamos National Laboratory strongly supports academic freedom and a researcher's right to publish; as an institution, however, the Laboratory does not endorse the viewpoint of a publication or guarantee its technical correctness. 


\title{
A LOGIC MODEL APPROACH TO CONCEPTUAL DESIGN OF A SCIENTIFIC/INDUSTRIAL COMPLEX
}

\author{
Terry F. Bott and Stephen W. Eisenhawer \\ Los Alamos National Laboratory \\ Los Alamos, New Mexico USA
}

\section{Introduction}

The development of a large-scale scientific/industrial complex is a difficult undertaking. At the conceptual design stage a number of issues must be carefully resolved for the project to be successful. A few of these questions include:

- What is the purpose of the complex?

- What capabilities are needed to express this purpose?

- How should the facilities that contain these capabilities be arranged and in what order should they be constructed?

- Which capabilities are most important in fulfilling the complex' mission and therefore what redundancy is needed?

Answers to these questions are needed whether the complex under consideration is planned or already exists and is undergoing a major restructuring. In the latter case additional constraints such as the need to maintain production, accommodate new missions and possible requirements to continue to use certain facilities may exist. The sheer size of the complexes under consideration here means that there will be many shareholders with a stake in the decision process and that the potential consequences of a poor decision could be severe. Different design options will exist in terms of scope and possibly in terms of schedule. Shareholders will value these options differently. Therefore a mechanism is needed to take into account the varying perspectives of the shareholders and to prioritize the options.

In this paper we show how the use of logic models can lead to more effective conceptual design. A deductive model is used to describe the relationships between the mission of a complex, the tasks that are the concrete expressions of the mission and the capabilities and resources that allow the mission to be carried out. This deductive logic model provides the formal basis for integrating these relationships into a conceptual design and provides a framework to express design options that are an essential part of the description of the complex. How is one to decide among the design options to reach the starting point for more detailed design? We show how a suitable decision model can be constructed using approximate reasoning (AR) (Zadeh 1976, Lopez de Mantaras 1990). AR uses forward-chaining inference models to emulate the types of reasoning used by experts.

The work described here was performed in support of a planning effort for updating the infrastructure for high explosives (HE) research and development (R\&D) at Los Alamos National Laboratory. HE research has been performed at Los Alamos since the Manhattan Project of World War II. The existing infrastructure was largely designed and built in the 1950's and 1960's to support a large R\&D effort in nuclear weapons during the Cold War. The rapid growth in the program coupled with the topography of the site - narrow mesas divided by deep arroyos, resulted in an infrastructure with facilities widely scattered over many square miles. At the time the increased costs associated with this situation were not considered important.

Following the cessation of nuclear testing and the end of the Cold War, the mission at Los Alamos shifted to one of maintaining nuclear deterrence through science-based methods. This change in emphasis has lead to concerns that the existing infrastructure might be inadequate to support the current mission of the Laboratory. Additionally, the costs of maintaining old facilities are increasing and have the potential to divert funds from facilities designed to provide new capabilities. Finally the separation of facilities is now seen as an impediment to encouraging the types of collaborative research needed in the new laboratory environment.

A committee of experts, the High Explosive Infrastructure Strategic Plan (HEISP) team, representing many areas of HE expertise, was formed in 1999 to produce a long-range concept for the HE infrastructure. The committee recognized that a major aspect of their responsibilities would include setting priorities for infrastructure upgrading, 


\section{DRAFT}

so that the capabilities posing the highest risk of disrupting the Laboratory mission would be candidates for early upgrade. In pursuit of these goals, the committee generated a large amount of predominantly qualitative information on HE capabilities and resources necessary to support the mission. The amount of information grew to become unmanageable and new techniques for organizing the data in a retrievable manner were necessary. In addition, they did not have an agreed upon approach to setting priorities.

The characteristics of this decision analysis problem made it suitable candidate for the Logic Evolved Decision (LED) model approach (Bott 2000, Eisenhawer 2002). LED was adapted to address setting priorities in the HE infrastructure vision development. In particular, it was used to systematically identify which HE capabilities and resources are needed to support institutional missions and to provide a consistent framework for evaluating the mission risk posed by these capabilities. Before presenting the LED results for the HEISP project, we describe the basic elements of an LED model in Section II.

\section{Overview of a Logic Evolved Decision Model}

The basic elements of an LED model are shown in Fig.1. We begin with some set of data that describes the system of interest. This information is a combination of quantitative and qualitative data. The data reside with a group of subject matter experts (SMEs). It is rare that any one person has access to all or even most of the relevant information. We use a deductive logic model to represent the system information that is elicited from the SMEs. The models are called process trees. Their basic mathematical structure is sufficiently powerful to represent physical systems such as weapons (Eisenhawer 1999), engineering processes (Bott 1999), sophisticated decision processes (Eisenhawer 2000) as well as the organization of a large scientific complex considered here.

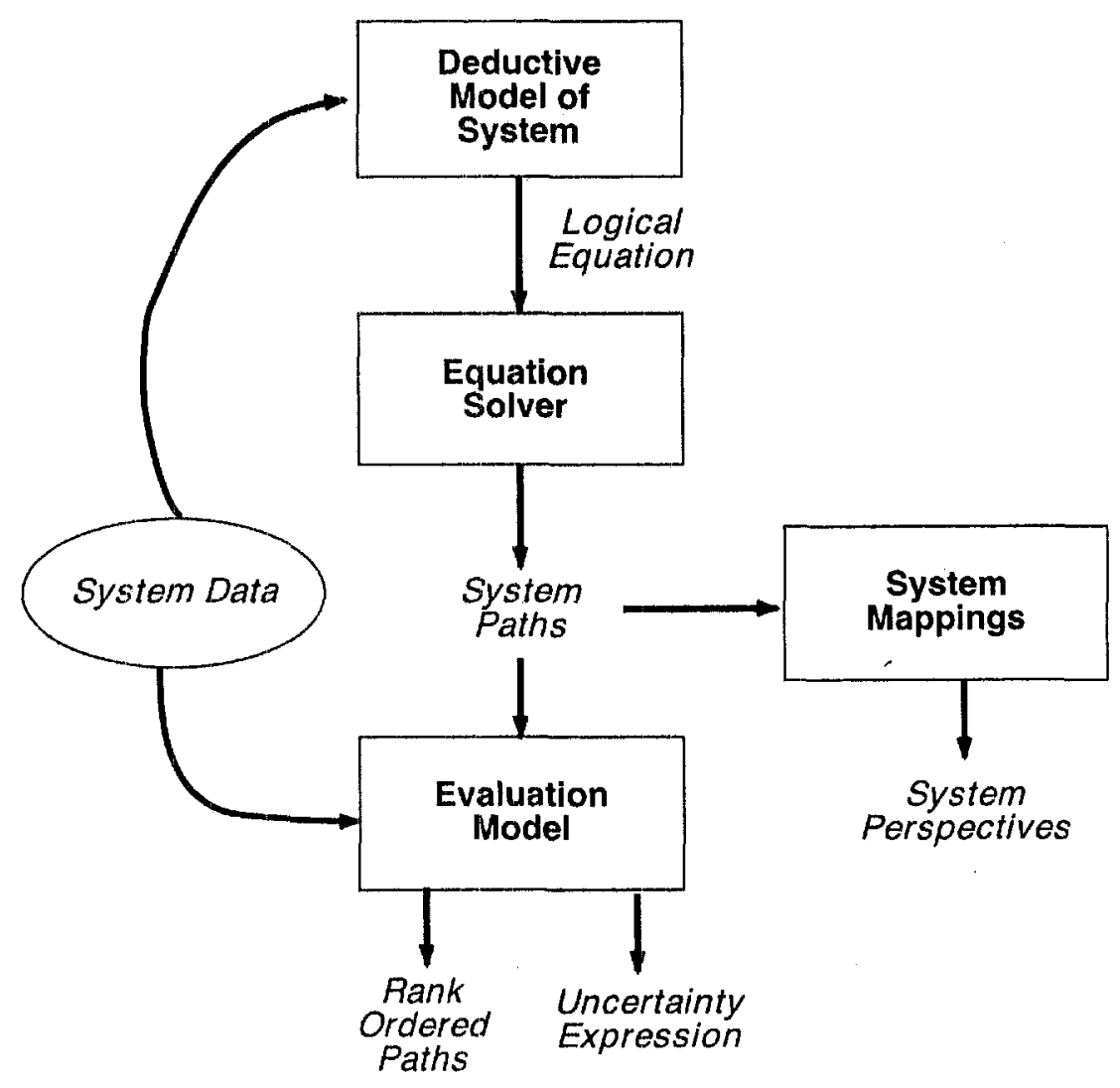

Figure 1. Overview of LED methodology. 


\section{DRAFT}

Consider the simple tree shown in Fig. 2. The top level here is the mission for the complex. Generally this will be known in advance. This mission is actualized by the performance of the three tasks - Tasks 1,2 and 3 . The use of an AND gate denotes that all three tasks must be performed for the complex to achieve its mission. Task 1 is determined to require two distinct capabilities A and B. Further analysis leads to the deduction that Capability B calls upon two resources. These resources may be facilities or more specifically a particular piece of equipment. The level of detail can vary depending upon the amount of information needed and can be expanded easily by adding new levels or elements to the tree. We have left Capability $\mathrm{A}$ as an undeveloped event, denoting that further detail will be added later. This is also true for Task 2. We use an OR gate to indicate that Task 3 has two alternatives - Task $3 \mathrm{~A}$ and Task 3B. Note that both of these tasks use Capability C. However Task $3 \mathrm{~A}$ also requires Capability $\mathrm{A}-$ associated earlier with Task 1, while Task 3B introduces the new Capability D. The flexibility to extend the tree as needed is clear; very large systems can be represented using this methodology.

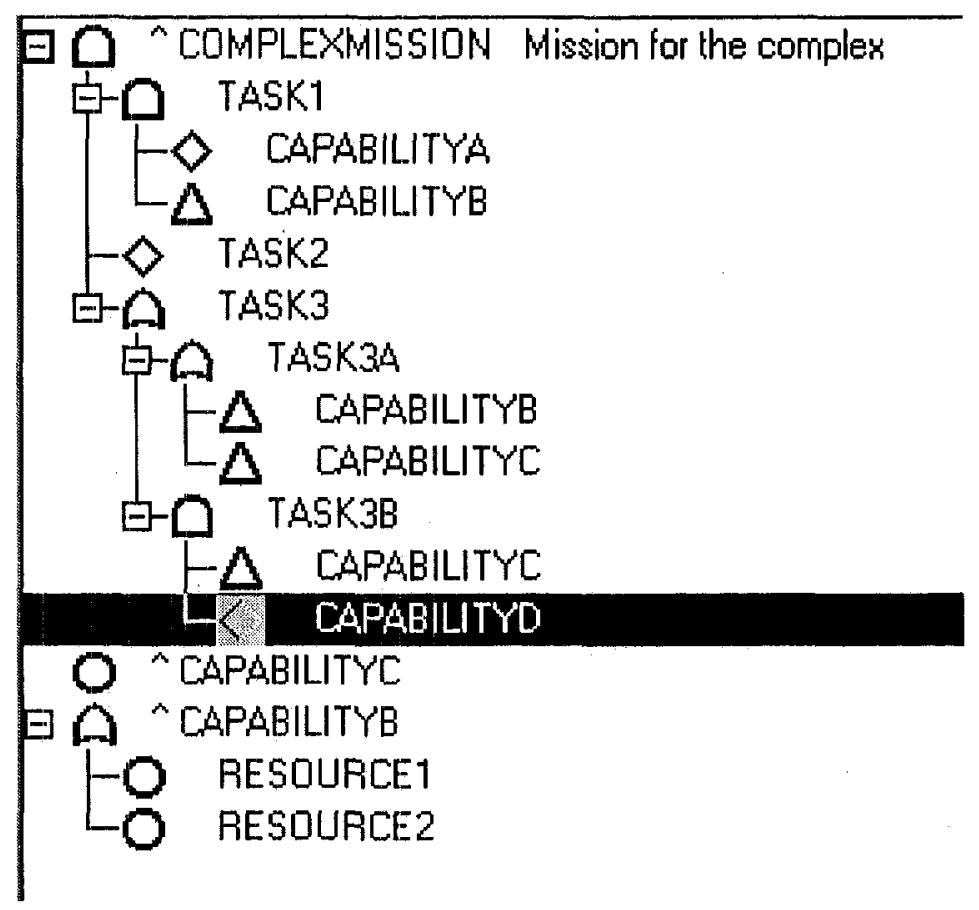

Figure 2. Simple process tree for a complex.

The process tree of Fig. 2 is a logical equation that can be solved. The inclusion of alternatives at various points in a tree leads to multiple solutions. The two solutions to this tree are

Complex Mission, Task1, Capability A, Capability B, Resource 1, Resource 2, Task 2, Task 3, Task 3A Capability B, Resource 1, Resource 2, Capability C

Complex Mission, Task1, Capability A, Capability B, Resource 1, Resource 2, Task 2, Task 3, Task 3B, Capability C, Capability $D$

Each solution is a path through the tree. By convention, a path begins at the top level and then grows by successive substitution of the appropriate gate inputs. A path is a complete description of a possible relationship between the basic elements of the system, here Resources 1 and 2 (as well as any undeveloped tasks and capabilities), and the mission of the complex. Note that in this example each path contains Capability C. 


\section{DRAFT}

The set of paths can be used to explore relationships in the tree. We call these relationships mappings. For example, the paths can be filtered to find all of the capabilities and resources related to a specific task or subtask.

Alternatively, one can produce a mapping between a single resource and all of the tasks that it supports. Logical proximity in a collection of paths may also indicate certain physical relationships that should be optimized in the complex design. These mappings are illustrated schematically in Fig. 3. We will illustrate the value of mappings in the discussion of the HEIS process tree below.

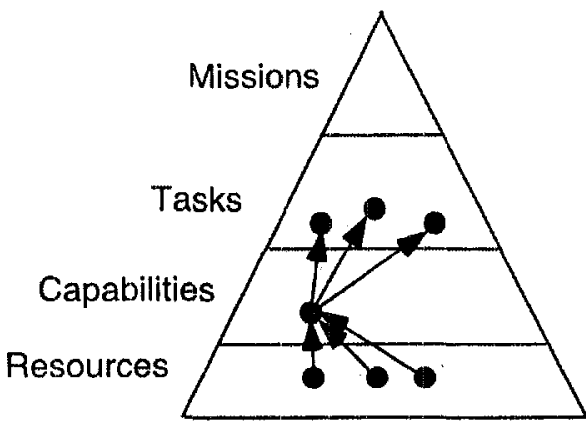

(A)

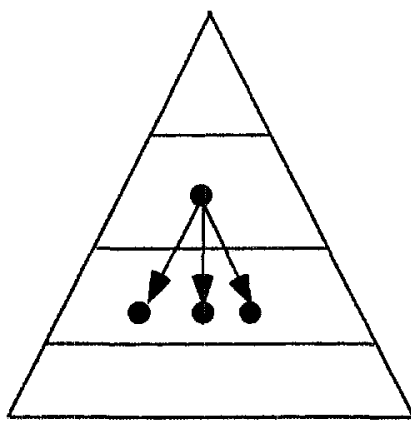

(B)

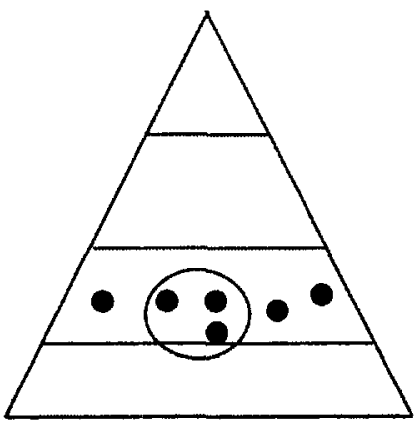

(C)

Figure. 3. Mappings from solution of the process tree. (A) capability to task, (B) task to capabilities, (C) logical proximity.

The set of systern paths is the input to the evaluation model. They are the alternatives or options to be evaluated. In LED we use approximate reasoning to perform this evaluation. Th reader may not be familiar with the concepts of AR so we provide a very short introduction here; greater detail is given in (Eisenhawer 2000).

\section{Operation of an AR Model}

There are three basic operations to be carried out to perform the sequence of evaluations shown in Fig. 4:

(1) conversion of the input information on the paths describing the complex into the proper form,

(2) solution of the forward-chaining inferential chain, and

(3) translation of the output into metrics to bin and order the upgrade possibilities. These steps correspond to the circled numbers in Fig. 4.

In this paper we will deal mainly with the first two of these.

Step 1: Conversion of the input. The data to be considered can be either quantitative (Circle 1a in Fig. 3) or qualitative (Circle $1 \mathrm{~b}$ ); both will be converted into the same internal form. In the model here all of the input variables are qualitative. AR models represent variables as linguistics-natural language expressions. Linguistic variables are particularly well suited for the representation of qualitative data.

The use of a linguistic variable can best be understood with an example. An important factor in assessing the risk associated with a capability is its current adequacy, $A_{c}$. We chose to represent $A_{c}$ linguistically with the following descriptors: inadequate, marginal, nominal and superior. These descriptors are referred to as the universe of discourse, $U=\{$ Inadequate, Marginal, Nominal, Superior $\}$. The elements in a universe of discourse are treated as fuzzy sets (Ross 1995, Klir 1995). A linguistic variable has associated with it a degree of membership vector, D, that defines to what extent it belongs to each set. The assignment of $D\left(A_{c}\right)$ is done by interpreting the expert elicitation.

For example, if it was judged that the capability adequacy was "clearly superior" then $D\left(A_{c}\right)=[0,0,0,1]$. If the judgment was that the adequacy was "somewhere between marginal and superior" and "closer to marginal" then one possible $D$-vector could be $D\left(A_{c}\right)=[0,6, .3, .2]$. This situation could arise when attempting to represent the 


\section{DRAFT}

opinions of multiple experts or when an expert has several different considerations that he wishes to take into consideration. ${ }^{1}$ Note also that there is no requirement that a $D$-vector sum to 1.0 ; degrees of membership in fuzzy sets are not probabilities.

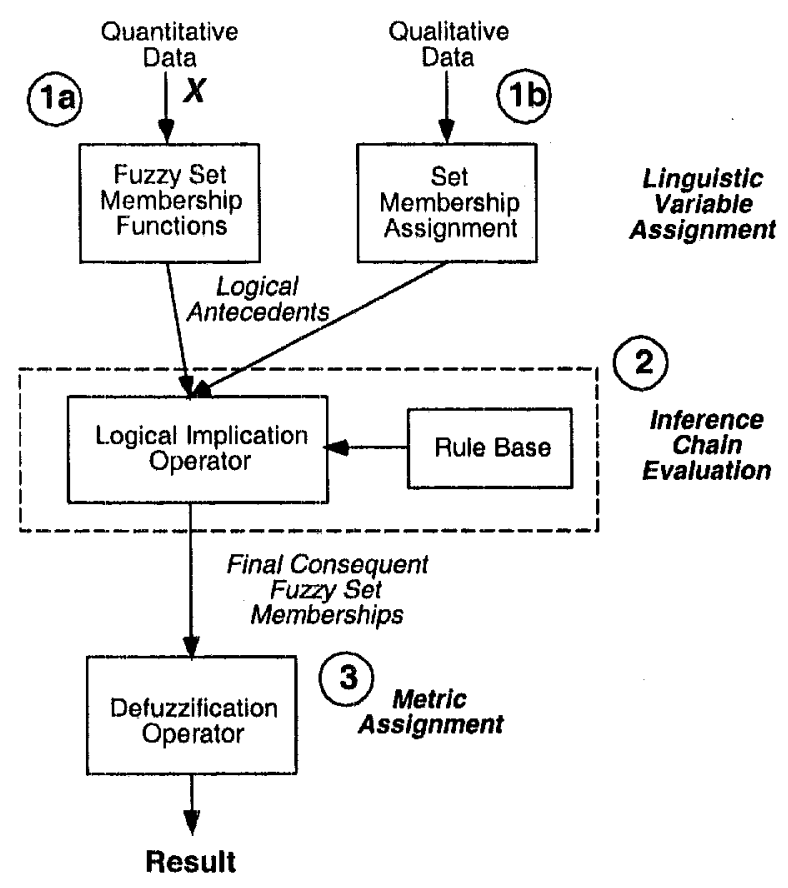

Figure. 4. Overview of the operation of an AR model.

Step 2: Solution of the inference chain. The fundamental logical operation in AR is implication, which is performed at each inference node in the model. A node has multiple inputs-the antecedents, $\mathrm{A}$-and a single output-the consequent, $\mathrm{C}$-all with their corresponding universes of discourse. For simplicity, consider the case where there are two elements in $A,\{\alpha, \beta\}$. The inference drawn is of the form:

" $\alpha$ is $\alpha_{1}$ and $\beta$ is $\beta_{\mathrm{j}}$, AND IF $\alpha_{1}$ and $\beta_{\mathrm{j}}$ THEN $\mathrm{C}_{\mathrm{k}}$ THEREFORE $\mathrm{C}_{\mathrm{k}}$ ",

where $\mathrm{i}, \mathrm{j}$, and $\mathrm{k}$ refer to particular elements in the respective universes of discourse. This statement is a tautology, hence the justification

for the use of the word "THEREFORE." The assignment of $\mathrm{k}$ given $\mathrm{i}$ and $\mathrm{j}$ is done with a rule base. This rule base describes how a combination of antecedent fuzzy sets implies a particular set in $U(C)$.

To make this concrete, consider Fig. 5. We propose to infer Conditional Current Risk, $R_{c-c o n d}$ from $A_{c}$ and some measure of the importance of the capability I, based upon the tasks it supports. For importance we use the linguistics $\mathrm{U}(\mathrm{I})=\{$ Ideal, Prudent, Necessary, Critical $\}$. "Ideal" suggests that there is little or no reliance on the capability for the task of concern. The risk is understood to be conditional because there is no consideration at this stage of

whether an alternative capability exists. We must also assign linguistics for risk $-U\left(R_{c-c o n d}\right)=\{$ Low, Moderate, High, Very High\}. Risk is normally expressed as a function of likelihood and consequence. Here we assert that the less adequate the capability, the more likely that the consequence, measured here by importance, will occur. The rule base for this node is given in Table 1. The shaded entry is the rule:

\footnotetext{
${ }^{1}$ This is referred to as hedging and is a very important feature of using a fuzzy set representation for linguistic variables.
} 


\section{DRAFT}

If $A_{c}$ is MARGINAL AND I is NECESSARY THEN $R_{c-c o n d}$ is HIGH.

Recall that both antecedents are represented internally as D-vectors, and in general, these vectors will have more than one non-zero entry. This means that multiple rules, and therefore assignments of $\mathrm{R}_{c \text {-cond }}$, are occurring simultaneously. A consistent resolution of this situation is obtained using the max-min rule (Ross 1995). This can be expressed as

$$
\mu_{\Re}=\underset{\forall(n, m) \rightarrow \Re}{\operatorname{Max}}\left(\operatorname{Min}\left(\kappa_{n}, \sigma_{m}\right)\right) .
$$

In this formula, $\kappa_{n}$ and $\sigma_{m}$ are elements $n$ and $m$ of fuzzy input membership vectors $\kappa$ and $\sigma$, and $\Re$ is a particular element output by the rule. For example, assume that $D\left(A_{c}\right)=[.3, .7,0,0]$-the capability adequacy has no membership in the sets "Nominal" or "Superior" and non-zero membership in "Marginal" and "Inadequate" and $\mathrm{D}(\mathrm{I})=[0, .2, .8, .2]$, corresponding to non-zero membership in "Prudent", "Necessary and "Critical." Applying the max-min operator to these $D$-vectors yields $D\left(R_{c-c o n d}\right)=[0,2, .7,3]$. A natural language interpretation of this vector is that $R_{c-c o n d}$ is between moderate and very high with the preponderance of evidence suggesting the risk is high. Of course current risk should taken into account whether alternatives exist. If they do then our judgment of current risk will be affected. This consideration can be taken into account by chaining $\mathrm{R}_{\mathrm{c} \text {-cond }}$ with a linguistic variable describing the efficacy of the alternatives in a second inference node. ${ }^{2}$

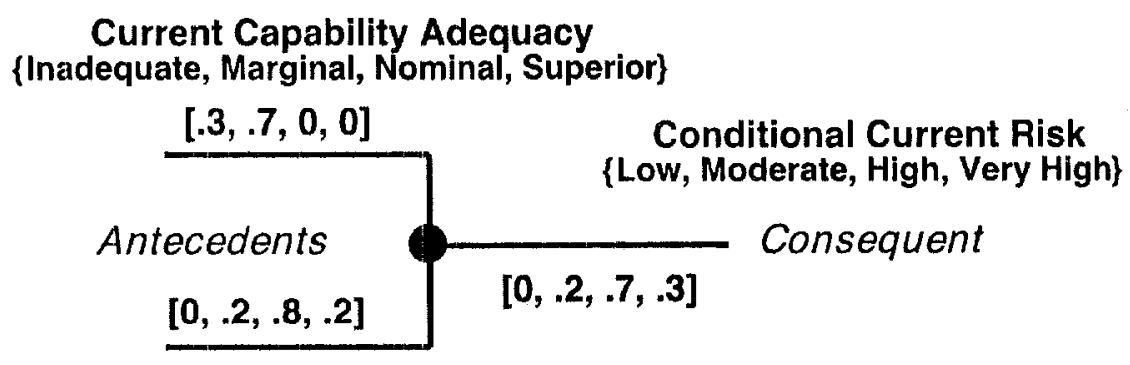

Capability Importance
\{Very Ideal, Prudent, Necessary, Critical\}

Figure. 5. Inference node for assessing conditional current risk.

Table 1. Rule Base for Conditional Current Risk, $\mathbf{R}_{\mathrm{c} \text {-cond }}$

\begin{tabular}{|c|c|c|c|c|c|}
\hline \multirow{4}{*}{} & \multicolumn{5}{|c|}{ Task Importance, I } \\
\cline { 2 - 6 } & & Ideal & Prudent & Necessary & Critical \\
\cline { 2 - 6 } & Superior & Low & Low & Low & Low \\
\cline { 2 - 6 } & Nominal & Low & Low & Low & Moderate \\
\cline { 2 - 6 } & Marginal & Low & Moderate & High & Very High \\
\cline { 2 - 6 } & Inadequate & Moderate & High & Very High & Very High \\
\hline
\end{tabular}

\footnotetext{
${ }^{2}$ In the actual HEISP evaluation model the three linguistic variables, current adequacy, importance and alternatives are the antecedents in a single three-level rule base used to infer current risk.
} 


\section{DRAFT}

Step 3: Translation of the output into metrics. The result of a set of forward-chained inferences is a single consequent and one D-vector. In our model (see Fig. 11), this will be "Mission Risk", $R_{m}$ with the same universe of discourse as $\mathrm{R}_{\mathrm{c} \text {-cond }}$. To use $\mathrm{R}_{\mathrm{m}}$ as our metric for ranking capabilities for upgrading, it must be converted to a numerical value that reflects the meaning of the $\mathrm{D}$-vector. This process is called defuzzification. We have discussed defuzzification in the context of an AR decision model at length in a previous paper (Eisenhawer 2000) and suggest here a very simple procedure to illustrate the principle. Logically, the most concern will be given to capabilities where the risk has some degree of membership in "Very High" as in the example above. The greater the degree of membership the higher the concern. Therefore we can use membership in "Very High" as the metric to rank order capabilities. The results presented below for the HEIS complex will use this metric.

\section{Deductive Model for HE Infrastructure}

A simplified version of the process tree for HEIS is shown in Fig. 6. It follows the basic structure discussed for Fig. 2. We chose the end state of interest to be "Ability to support the Laboratory missions". ${ }^{3}$ The missions were deduced from the official Laboratory mission statement:

- Ensure the safety and reliability of the U.S. Nuclear deterrent.

- Reduce the global threat of weapons of mass destruction.

- Solve national problems in energy, environment, infrastructure and health security.

Our focus was on tasks associated primarily with nuclear weapons and of course high explosives; the other missions and tasks were not considered in detail. These are grouped under the element "NON NUCLEARTASKS" (denoted by the $A$ in Fig. 6). The next step is to logically develop possible ways to perform the nuclear weapons task - NUCLEAR TASKS. Four primary nuclear weapon tasks were identified

- Weapon system support: providing necessary services to the Department of Defense for nuclear weapons in the custody of the Armed forces.

- Existing stockpile support: to be discussed in more detail.

- Weapon development: development of new nuclear weapons. This activity is not a significant influence on HE infrastructure.

- Weapons complex environmental restoration: activities related to the clean-up of the weapons complex.

Each of these is developed in extensive detail in the full tree.

A closer look at the existing stockpile support task $(B)$ shows five subtasks. One of these, surveillance program $(C)$ is developed in more detail. Nuclear weapons are the subject of an extensive surveillance program. One aspect of this involves the characterization of high explosives $(D)$ to confirm that the performance remains within specification. Characterization is a capability. Figure 7 shows the development of the sub-tree associated with this capability. The HE is characterized according to five sets of properties, Chemical, Microstructure ( $A$ in Fig. 7 ), Explosive, Mechanical and Thermal. Using microstructure as the example we deduce needs for a sample acquisition and preparation capabilities. These are prerequisites for the actual morphology characterization capability $(B)$. At this level, resources such as various forms of microscopy begin to appear. In some cases a resource is already directly related to a facility such as the Los Alamos Neutron Science Center (C) where neutron diffraction studies are performed.

\footnotetext{
${ }^{3}$ Note that the form of the HEIS process tree is not unique because different organizing principles lead to different structures for the tree.
} 


\section{DRAFT}

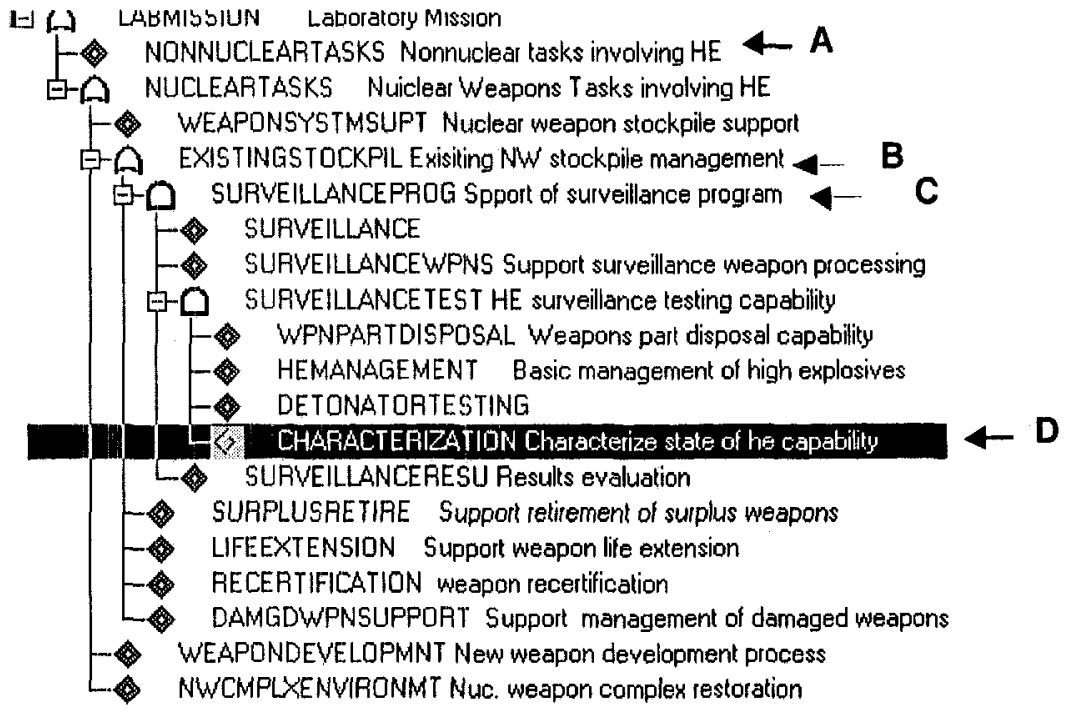

Figure. 6. Top levels of the HEIS process tree. The double diamond symbol indicates that in the complete tree these branches have been developed.

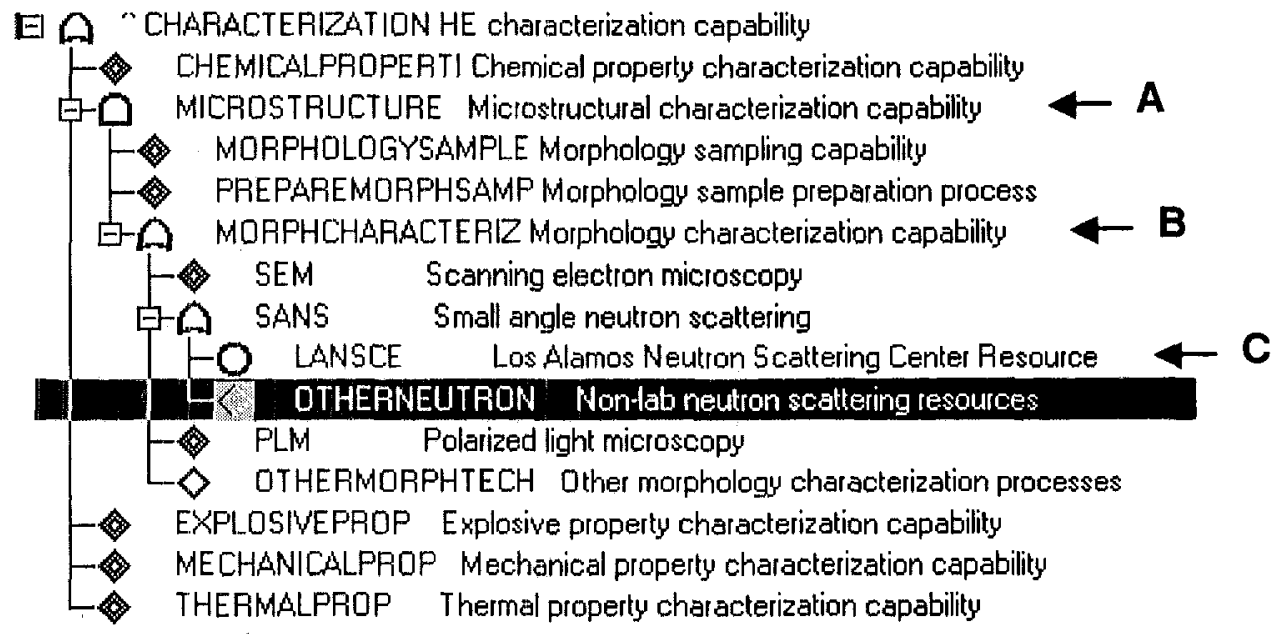

Figure 7. Sub-tree for representation of HE characterization capability.

Several aspects of the HEIS tree are characteristic of logic models for a scientific/industrial complex. This structure occurs because a limited number of missions lead to a larger number of tasks, some of which are shared between missions. The tasks are supported by an even larger number of capabilities, with greater commonality at this level. Finally, the capabilities are supported by many resources. At the resource level there are many more possibilities than at the higher levels. This is because there are often several alternative resources that could be used to provide a given capability. The tasks and capabilities required to support the mission are more specific and generally are unique. The Laboratory missions are supported by high-level tasks. These tasks in many cases correspond directly to existing programs. In other cases the tasks are supported by parts of, or the entirety of, several programs. In a few cases, several tasks combine to form an existing program. How does this mismatch between existing programs and the tasks in the process tree arise? The tree tasks are logically derived from the mission. However the evolution of a program is affected by historical, organizational, financial and political factors as well. 


\section{DRAFT}

\section{Infrastructure Mappings}

After solving the complete tree, we first produced a series of mappings to help the SMEs understand the logical structure of the HE infrastructure. Forty-four distinct capabilities were identified. These could be grouped into seven sets. Each set represents a subcomplex as described in Table II. The number of capabilities in each subcomplex varies according to the level of detail needed to identify the important resources. Independently the experts had spent time considering how capabilities could be related to one another. By combining several of the subcomplexes we were able to confirm the relationships suggested by the experts. One way to express these relationships is in terms of a Venn diagram as shown in Fig. 8. This diagram served to define "clusters" of facilities. Based upon these clusters and the previously mentioned site topology, new co-locations of resources and capabilities were proposed.

\section{Table II. Subcomplexes identified for HE infrastructure}

\begin{tabular}{|c|c|l|}
\hline $\begin{array}{c}\text { Infrastructure } \\
\text { Subcomplex }\end{array}$ & $\begin{array}{c}\text { Number of } \\
\text { Capabilities }\end{array}$ & \multicolumn{1}{|c|}{ Brief Description } \\
\hline Production & 12 & $\begin{array}{l}\text { Synthesis and formulation of explosives. Explosive molding, pressing } \\
\text { and machining operations. }\end{array}$ \\
\hline Detonators & 4 & Detonator design, production testing and research. \\
\hline Assembly & 8 & $\begin{array}{l}\text { Storage, assembly and disposal of HE devices and weapon } \\
\text { components. }\end{array}$ \\
\hline HE Management & 4 & HE receiving, transport, storage and waste management. \\
\hline Explosive Firing & 10 & Explosive test design and operations including diagnostics. \\
\hline HE Physics & 4 & HE environments, damage states and aging \\
\hline HE Chemistry & 2 & Organic chemistry research and HE characterization \\
\hline
\end{tabular}

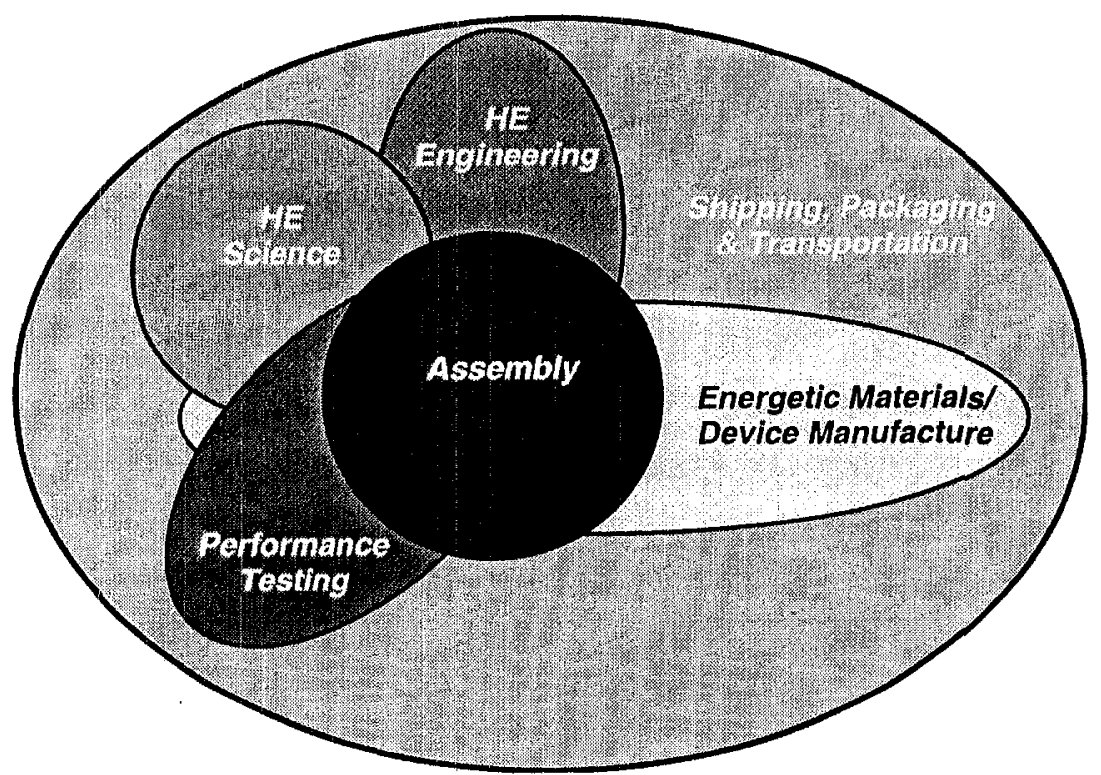

Figure 8. Venn diagram illustrating the logical relationships among capability clusters. 


\section{DRAFT}

Mapping from a particular task to the capabilities that support it is useful for planning what types of facilities and equipment are needed to support a task. This type of mapping is shown conceptually in Figure 9 for a specific task Recertification of weapons in the stockpile ${ }^{4}$. In this case the logic model showed that there were 32 capabilities needed for weapon recertification. These capabilities were associated with five of the seven subcomplexes. This type of mapping provides a means for optimizing the construction of resources to provide the most capability within space, environmental, cost and other constraints.

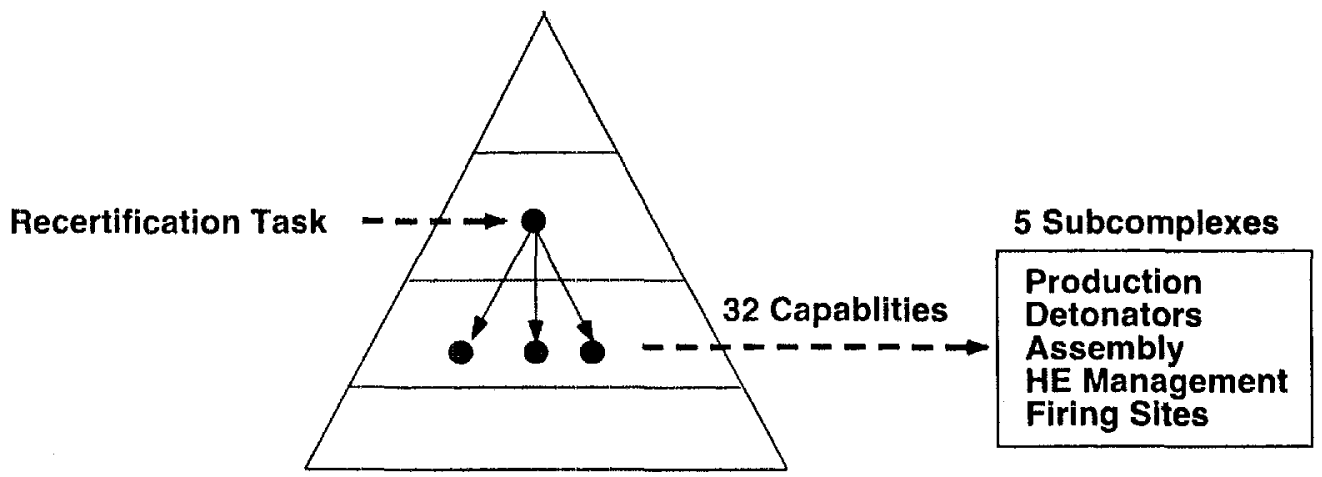

Figure 9. Task to capability mapping.

If one maps from a specific capability or group of capabilities to the missions it supports then other factors are emphasized. This type of mapping is shown conceptually in Figure 10. The capability here is HE Plant. This cluster does not correspond directly to any of the logical subcomplexes in the process tree but is instead a combination of some capabilities from HE Management and Production. This particular cluster was of interest to the experts. The mapping was obtained by filtering the paths for these capabilities and extracting the corresponding set of subtasks. It is useful in illustrating the importance of a capability. A capability that is widely utilized by many important missions is important. This type of mapping can be used to explore the possibility of new missions that the existing infrastructure is able to support with little or no need for additional capabilities. The mapping could also be performed from the resource level to any higher level as well. Note that these mapping are obtained from simple mathematical operations on the collection of solution paths. Therefore it is very easy to explore the relationships between many possible capability configurations and collections of tasks in a systematic way.

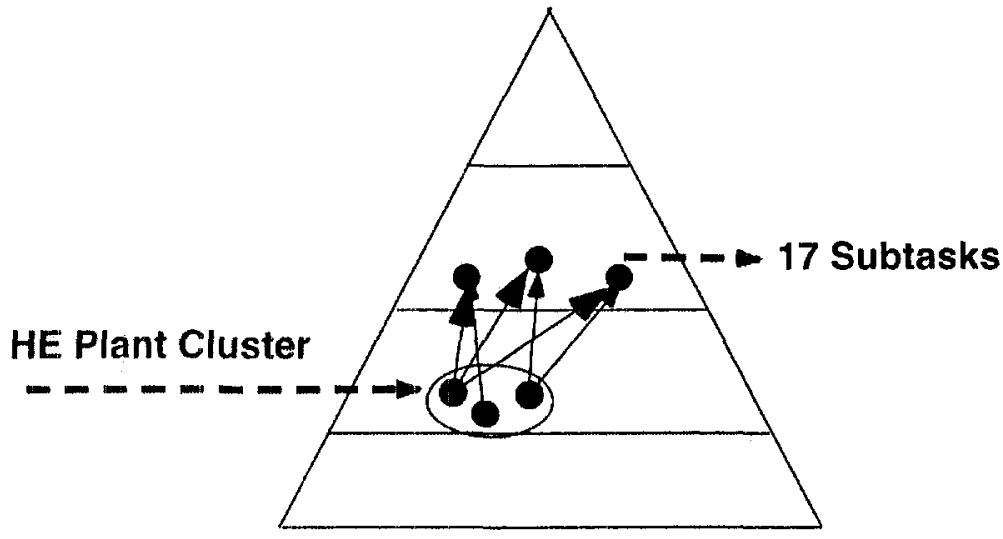

Figure 10. Capability to task mapping. ${ }^{4}$ Recertification is the annual process of affirming that nuclear weapons are safe, reliable and will
perform as specified. 


\section{DRAFT}

\section{Evaluation Model}

As noted earlier, the metric chosen to rank order the capabilities is programmatic risk. Programmatic risk includes the likelihood of a capability being unable to support a task, the importance of that task to a given mission, and the importance of that mission. We showed earlier how a component of this risk, conditional current risk, can be inferred using linguistic variables and an implication rule base. The complete set of factors used in the evaluation of programmatic risk were determined in discussions with the HEISP team. In these discussion they expressed their ideas about the considerations that would be important in setting priorities for facility replacement.

The AR model designed to use these factors is shown in Fig. 11. Each node is evaluated according to the techniques described previously. Mission risk depends both upon the tasks that support it and the capabilities that service these tasks. For each task-capability pair a mission risk is inferred based upon the importance of the task and the future risk for the capability (Node A in Figure 11). The task importance was elicited from the Team experts. Future capability risk (Node B) is the result of the inferential chain used to evaluate current risk (Node C) as discussed previously, and a prognosis submodule that projects the change in risk. For the base case evaluation no facility upgrades are considered in the prognosis inference. The prognosis (Node D) is inferred from expert assessments of technical prognosis, which takes into account such factors as obsolescence and operational prognosis which includes such factors as reliability, parts availability, etc.

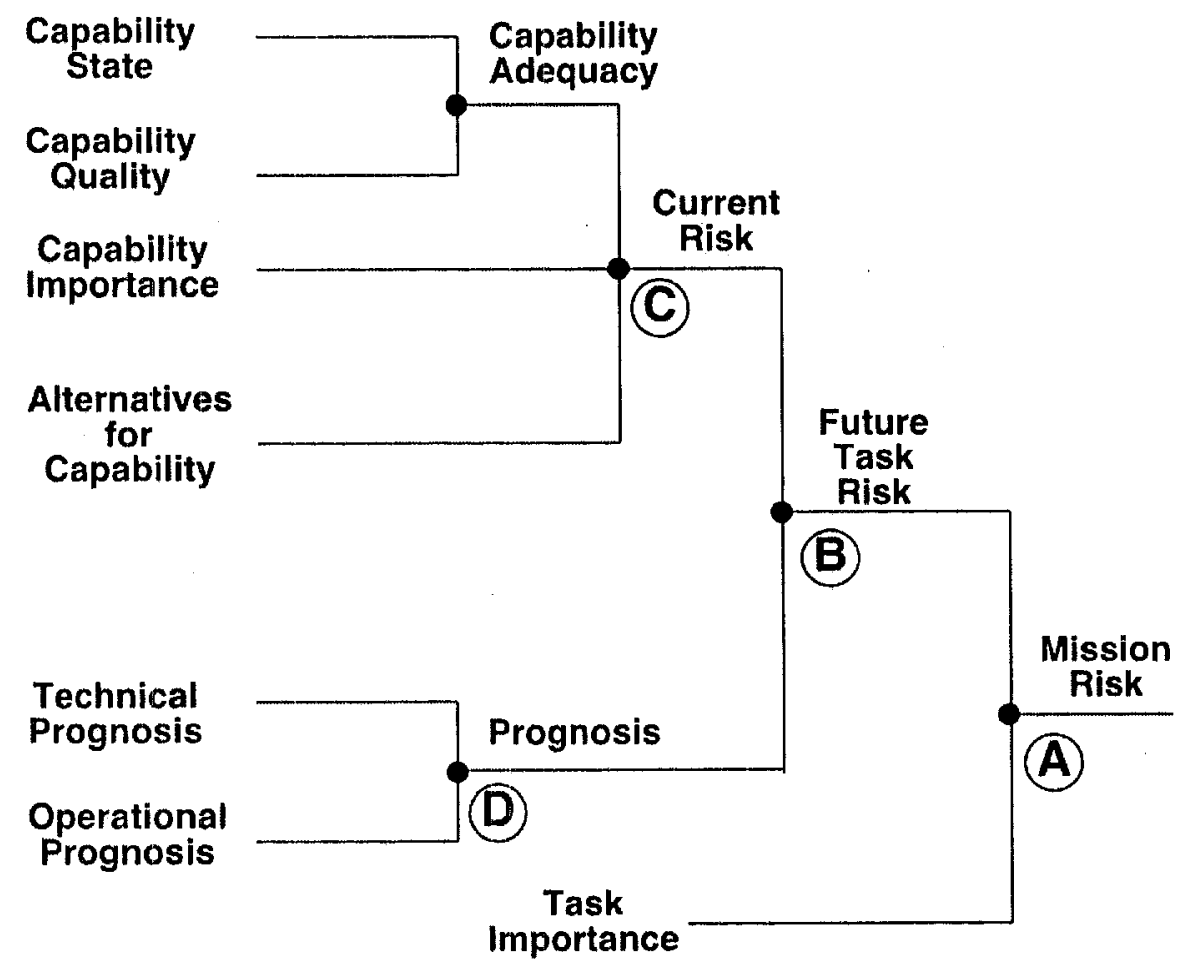

Figure 11. AR model for inferring programmatic risk.

An example of the risk evaluation is shown in Fig. 12. Here we plot the metric, degree of membership in the "Very High" set for the principal Laboratory nuclear weapons tasks. The set of twenty-seven capabilities here is for a conceptual subcomplex that would provide support for firing site operations. One can see at a glance which capabilities are believed to pose high risk to one or more Laboratory tasks, and the relative degree of this belief. Of course this same type analysis can be carried out at the mission level. Note that by using the mapping and risk evaluation analyses together it is possible to explore various plans for infrastructure upgrades in a systematic manner. 


\section{DRAFT}

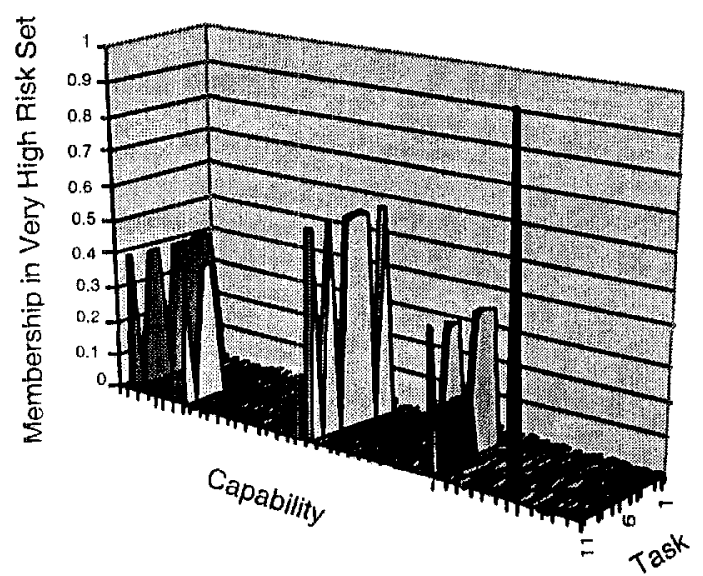

Figure 12. Programmatic risk for multiple tasks from supporting capability set.

\section{Conclusions}

In this paper we have demonstrated a general approach to systematically and reproducibly generating capability sets for scientific and industrial complexes based on the missions of the complex. In this particular application, an existing infrastructure was analyzed to identify the capabilities that posed the highest risk to meeting the institutional mission. We believe LED would be especially useful in systematically identifying the capability sets needed in a new complex or facility. The process tree provides a platform for deducing what capabilities may be needed for innovative missions and provides a means for identifying a range of possible approaches to providing the necessary resources. AR models are well suited to evaluating the possibilities generated by the process tree. They provide a consistent, traceable and robust way to make informed decisions when much of the relevant information is qualitative in nature. This is exactly the case in conceptual design.

The results shown here were briefed to high-level management at Los Alamos. The insights gained through this analysis formed a major part of the recommendations made to these managers. They responded favorably to the analysis because it demonstrated a serious attempt to be systematic and thorough in proposing facility upgrades. It also provided them with documentation of the reasoning for the recommendations. Members of the steering committee also rallied behind the recommendations. Although the potential for parochialism was strong, the open and fair decision making afforded by this analysis reduced the concerns of all parties and a consensus was reached on the priorities. These practical results confirm our belief that LED is an excellent tool for long range planning.

\section{Acknowledgment}

The authors wish to thank Dr. Joseph V. Repa for financial support, advice and encouragement in the development of the Logic Evolved Decision model methodology. We would also like to thank the members of the HEISP Committee, and in particular the chairman, Dr. Paul Cunningham, as well as many other subject matter experts at Los Alamos for their help in performing this work.

\section{References}

(Bott 1999) T. F. Bott, S. W. Eisenhawer and S. F. Agnew, "Evaluation of High-Level Waste Pretreatment Process with an Approximate Reasoning Model," Proc. Waste Management Symposium, 1999 Feb.

(Bott 2000) T. F. Bott, S. W. Eisenhawer, "An Approach to Assessing the Need for High Explosives Replacement in Aging Nuclear Weapons," $27^{\text {th }}$ International Pyrotechnics Seminar, 2000 Jul. 


\section{DRAFT}

(Eisenhawer 1999) S. W. Eisenhawer, T. F. Bott, "Application of Approximate Reasoning to Safety Analysis," Proc, International System Safety Conference, 1999 Aug, pp 374-382.

(Eisenhawer 2000) S. W. Eisenhawer, T. F. Bott, R. E. Smith, "An Approximate Reasoning-Based Method for Screening High-L_evel-Waste Tanks for Flammable Gas," Nuclear Technology, Vol. 130, 2000 Jun, pp 351-361.

(Eisenhawer 2002) S. W. Eisenhawer, T. F. Bott, J. W. Jackson, "Prioritizing the Purchase of Spare Parts Using an Approximate Reasoning Model," Proc. International Symposium on Product Quality and Integrity, January 2002.

(Klir 1995) G. J. Klir, B. Yuan, Fuzzy Sets and Fuzzy Logic, 1995, Prentice-Hall.

(Lopez de Mantaras 1990) R. Lopez de Mantaras, Approximate Reasoning Models, 1990, Ellis Howrood.

(Ross 1995) J. Ross, Fuzzy Logic with Engineering Applications, 1995, McGraw-Hill.

(Zadeh 1976) L. Zadeh, "A Theory of Approximate Reasoning," in Machine Intelligence, J. Hayes, D. Michie, L. Mikulich (Ed.), 1976, Halstead Press. 